\begin{tabular}{|c|c|}
\hline Title & A $n$ analytic solution of steady Stokes flow on a rotating polar cap \\
\hline Author(s) & Kitauchi, Hideaki; Ikeda, Motoy oshi \\
\hline Citation & $\begin{array}{l}\text { Fluid Dynamics Research, 41(4), } 045505 \\
\text { https://doi.org/10.1088/0169-5983/41/4045505 }\end{array}$ \\
\hline Issue Date & 2009-08 \\
\hline Doc URL & http:/hdl.handle.net/2115/39627 \\
\hline Rights & $\begin{array}{l}\text { This is an author-created, un-copy edited version of an article accepted for publication in Fluid Dynamics Research. IOP } \\
\text { Publishing Ltd is not responsible for any errors or omissions in this version of the manuscript or any version derived } \\
\text { from it. The definitive publisher authenticated version is available online at } 10.1088 / 0169-5983 / 41 / 4 / 045505 \text {. }\end{array}$ \\
\hline Type & article (author version) \\
\hline File Information & FDR41-4_045505.pdf \\
\hline
\end{tabular}

Instructions for use 


\title{
An analytic solution of steady Stokes flow on a rotating polar cap
}

\author{
Hideaki Kitauchi ${ }^{\mathrm{a}}{ }^{*}$ and Motoyoshi Ikeda ${ }^{\mathrm{b}}$ \\ ${ }^{a}$ Frontier Research Center for Global Change, Japan Agency for Marine-Earth \\ Science and Technology, Yokohama, Kanagawa 236-0001, Japan \\ ${ }^{\mathrm{b}}$ Faculty of Environmental Earth Science, Hokkaido University, \\ Sapporo, Hokkaido 060-0810, Japan
}

Received 26 April 2007; revised 9 July 2008; accepted 20 January 2009

Communicated by Y. Hayashi

\begin{abstract}
An analytic solution of two-dimensional, steady, linear, viscous flow on a polar cap-polar region of a sphere which lies above (or below) a given plane normal to the rotation axis - rotating about its center is obtained. Inflow and outflow on the boundary of the polar cap drive the fluid motion. The solution of stream function is expressed as the Fourier series in longitudes and the associated Legendre functions of complex degrees in cosines of colatitudes. The fluid particles move almost along lines of constant latitudes, some circulate cyclonically and the others anticyclonically, in the geostrophic balance everywhere except near the north pole where the flow is relatively slow and the viscous force dominates over the Coriolis force. Our results support the approximation analysis and laboratory experiment studied by Imawaki and Takano (Deep-Sea Res. 21, 69-77, 1974).
\end{abstract}

Key words: Analytic solution; Stationary; Stokes flow; Rotation; Polar cap

\section{Introduction}

Flow, driven by inflow and outflow, on a circular basin (or polar cap) rotating about its center is a simple, fundamental model to study deep (not affected

* Corresponding author. Tel.: +81-45-778-5630; fax: +81-45-778-5706.

E-mail address: kitauchi@jamstec.go.jp (H. Kitauchi). 
by surface forcing, ex., wind stress or heating/cooling) circulations in, for example, the Norwegian Sea (Pratt, 1997) and the Arctic Ocean (Yang, 2005).

Among those studies, Imawaki and Takano (1974) investigated steady, linear, viscous, incompressible two-dimensional fluid motions, vertically integrated from the bottom to the surface of the ocean, on a rotating circular basin. The bottom is horizontal and the surface is free from stress due to wind or sea-ice drift. The flow is driven by an inflow and an outflow on the basin boundary. They obtained approximate solutions of the vorticity equation, that is a partial differential equation in longitude and latitude of the spherical coordinates on a polar cap. The solution of stream function is expressed as Fourier series in longitude, reducing the vorticity equation to a set of variable-coefficient ordinary differential equations in latitude. Then, they assumed that the solution is little affected by variations of latitudes appeared in those variable coefficients and replaced all the latitudes with some constant, the middle latitude between the basin center and boundary (we may call the middle of the zonal band), making the variable-coefficient ordinary differential equations those of constant coefficients. The solution is, therefore, expressed as the linear combination of exponential functions in latitude. They further confirmed their approximate solutions by the laboratory experiments. Their approach works fine and easily allows to include other effects (or terms) in their formulation, such as lateral and bottom frictions, so long as the variations of latitudes are

relatively small, that is, relatively narrow width of the zonal band of a polar cap (in their case, $20^{\circ}$ ).

This paper revisits the problem done by Imawaki and Takano (1974). Instead of replacing all the latitudes in the coefficients with some constant, we analytically solve the vorticity equation without the above assumption. Although we obtain an analytic solution similar to the approximate solution obtained by Imawaki and Takano (1974), as you see later in this paper, our formulation is more general than theirs and allows the application to a basin having relatively wide zonal band. We also look into the balance of forces acting on fluid elements and the dependence of flow field upon non-dimensional physical parameter the horizontal Ekman number.

\section{Formulation}

We consider the motion of a fluid of constant density $\rho$ on a polar cap of radius $r$ rotating about its center with a constant angular velocity $\boldsymbol{\Omega}$ (see Fig. 1). We employ the spherical coordinates $(\vartheta, \varphi) \in\left[0, \vartheta_{\mathrm{b}}\right] \times[0,2 \pi)$ of radius $r$, where $\vartheta_{\mathrm{b}}$ is the colatitude of the boundary of the polar cap (or the spherical section). Although our configuration of the system is assumed to be flow around the north pole, our formulation is universal in both the poles - just change the sign 
of the angular velocity, $-\boldsymbol{\Omega}$, in case of flow around the south pole. The flow is assumed to be thin enough to neglect the motion perpendicular to the polar cap and slow enough to neglect the non-linear effects. Only the steady state is considered. There are an inflow and an outflow on the spherical section, which drive the fluid motion. The total inflow and outflow are same and constant in time. The equation of motion is written, in a frame rotating with the polar cap, as

$$
2 \boldsymbol{\Omega} \times \boldsymbol{u}=-\frac{1}{\rho} \nabla p+\nu \nabla^{2} \boldsymbol{u},
$$

and the continuity equation as

$$
\nabla \cdot \boldsymbol{u}=0
$$

where $\boldsymbol{u}(\vartheta, \varphi)=\left[u_{\vartheta}(\vartheta, \varphi), u_{\varphi}(\vartheta, \varphi)\right]$ is the two-dimensional velocity on the sphere, $p(\vartheta, \varphi)$ is the pressure, and $\nu$ is the constant kinematic viscosity of the fluid. The equation of motion (1) shows the balance between the Coriolis force, the pressure gradient force, and the viscous force. Taking the radial component of the curl of both the sides of Eq. (1) and using Eq. (2), we obtain the vorticity equation,

$$
-\beta u_{\vartheta}=\hat{\boldsymbol{r}} \cdot\left[\nabla \times\left(\nu \nabla^{2} \boldsymbol{u}\right)\right],
$$

where $\beta(\vartheta)=2|\boldsymbol{\Omega}| \sin \vartheta / r$ is the so-called $\beta$-term, and $\hat{\boldsymbol{r}}$ is the unit radius vector. Being quite small in polar regions compared to the other regions, the $\beta$-term still plays an important role; for the fluid motion would be the same as one without the rotation of the polar cap, if the $f$-plane approximation on the north pole is applied. The vorticity equation (3) shows that where the viscous force is negligible, the fluid particles move along lines of constant latitudes in the geostrophic balance. In other words, the viscous force makes the fluid particles cross lines of constant latitudes.

To solve Eqs. (2) and (3) we introduce the stream function $\psi(\vartheta, \varphi)$ so that the continuity equation (2) is satisfied,

$$
\boldsymbol{u}=\hat{\boldsymbol{r}} \times \nabla \psi,
$$

or

$$
u_{\vartheta}=-\frac{1}{r \sin \vartheta} \frac{\partial \psi}{\partial \varphi}, \quad u_{\varphi}=\frac{1}{r} \frac{\partial \psi}{\partial \vartheta} .
$$

Then, the vorticity equation (3) is rewritten as

$$
\left(\Delta_{\mathrm{H}}^{2}-E_{\mathrm{H}}^{-1} \frac{\partial}{\partial \varphi}\right) \psi(\vartheta, \varphi)=0,
$$

where $\Delta_{\mathrm{H}}$ is the horizontal Laplacian on a sphere,

$$
\Delta_{\mathrm{H}}=\frac{1}{\sin \vartheta} \frac{\partial}{\partial \vartheta}\left(\sin \vartheta \frac{\partial}{\partial \vartheta}\right)+\frac{1}{\sin ^{2} \vartheta} \frac{\partial^{2}}{\partial \varphi^{2}},
$$


and $E_{\mathrm{H}}$ is the horizontal Ekman number,

$$
E_{\mathrm{H}}=\frac{\nu}{2|\Omega| r^{2}} .
$$

We expand the stream function in terms of a Fourier series in longitudes,

$$
\psi(\vartheta, \varphi)=\sum_{m=-\infty}^{\infty} \hat{\psi}_{m}(\vartheta) e^{i m \varphi}
$$

where $\hat{\psi}_{m}(\vartheta)$ are the Fourier coefficients,

$$
\hat{\psi}_{m}(\vartheta)=\frac{1}{2 \pi} \int_{0}^{2 \pi} \psi(\vartheta, \varphi) e^{-i m \varphi} d \varphi \quad \text { for } m=0, \pm 1, \pm 2, \ldots
$$

Multiplying Eq. (5) by $e^{-i m \varphi}$ for an arbitrary integer $m$ and integrating over the longitudes $[0,2 \pi)$, we obtain a set of equations for the Fourier coefficients (cf. Courant and Hilbert, 1989, Ch. V §6.2, §9.1),

$$
\left(D_{m}^{2}-k_{m}^{4}\right) \hat{\psi}_{m}(\vartheta)=0 \quad \forall m
$$

that is,

$$
\left(D_{m}-k_{m}^{2}\right)\left(D_{m}+k_{m}^{2}\right) \hat{\psi}_{m}(\vartheta)=0 \quad \forall m .
$$

Here, the Laplacian operator $D_{m}$ is defined by

$$
D_{m}=\frac{1}{\sin \vartheta} \frac{d}{d \vartheta}\left(\sin \vartheta \frac{d}{d \vartheta}\right)-\frac{m^{2}}{\sin ^{2} \vartheta}
$$

and the constant parameter $k_{m}$ by $k_{m}^{4}=i m E_{\mathrm{H}}^{-1}$. The vorticity equation (3) is reduced to a set of the fourth-order ordinary differential equations (10) for the Fourier coefficients $\hat{\psi}_{m}$ over the colatitudes $\left(0, \vartheta_{\mathrm{b}}\right)$.

Before going further into our analysis, we briefly review that of Imawaki and Takano (1974), in which Eq. (10) is expanded as

$$
\begin{aligned}
& {\left[\frac{d^{4}}{d \vartheta^{4}}+\theta_{1} \frac{d^{3}}{d \vartheta^{3}}+\left(-m^{2} \theta_{2}+\theta_{3}\right)\right.} \frac{d^{2}}{d \vartheta^{2}}+\left(-m^{2} \theta_{4}+\theta_{5}\right) \frac{d}{d \vartheta} \\
&\left.+\left(m^{4} \theta_{6}-m^{2} \theta_{7}\right)-i m \kappa\right] \hat{\psi}_{m}(\vartheta)=0 \quad \forall m
\end{aligned}
$$

where the variable coefficients $\theta_{1}, \theta_{2}, \ldots, \theta_{7}$ and constant parameter $\kappa$ are, following their notations (cf. equations (6) and (7) in their paper), given by

$$
\begin{array}{lll}
\theta_{1}=\frac{2 \cos \vartheta}{\sin \vartheta}, & \theta_{2}=\frac{2}{\sin ^{2} \vartheta}, & \theta_{3}=-\left(1+\frac{1}{\sin ^{2} \vartheta}\right), \\
\theta_{4}=-\frac{2 \cos \vartheta}{\sin ^{3} \vartheta}, & \theta_{5}=\frac{\cos \vartheta}{\sin ^{3} \vartheta}, & \theta_{6}=\frac{1}{\sin ^{4} \vartheta}, \\
\theta_{7}=\frac{2+2 \cos ^{2} \vartheta}{\sin ^{4} \vartheta}, & \kappa=E_{\mathrm{H}}^{-1}=\frac{2|\Omega| r^{2}}{\nu} .
\end{array}
$$


They assumed that the solution is little affected by variations of colatitudes $\vartheta \in\left[0, \vartheta_{\mathrm{b}}\right]$ appeared in the coefficients $\theta_{1}, \theta_{2}, \ldots, \theta_{7}$ and replaced all of them with some constant colatitude $\vartheta_{\mathrm{c}}$, the middle between the pole and boundary (we may call the middle of the zonal band), $\vartheta_{\mathrm{c}}=\vartheta_{\mathrm{b}} / 2$, making the variablecoefficient ordinary differential equations (12) the constant-coefficient ordinary differential equations. Then, the solution is assumed to be proportional to the exponential function, $\hat{\psi}_{m}(\vartheta) \propto e^{t^{m}} \vartheta$, for every order $m$, the fourth-order ordinary differential equation (12) determines the four complex numbers $t^{m}=$ $t_{1}^{m}, t_{2}^{m}, t_{3}^{m}, t_{4}^{m}$ as roots of the quartic equation,

$$
\begin{aligned}
t^{4}+\theta_{1} t^{3}+\left(-m^{2} \theta_{2}+\theta_{3}\right) t^{2}+\left(-m^{2} \theta_{4}+\theta_{5}\right) t & \\
& +\left(m^{4} \theta_{6}-m^{2} \theta_{7}\right)-i m \kappa=0 \quad \forall m .
\end{aligned}
$$

The solution is therefore, in general, expressed as the linear combination of the four exponential functions,

$$
\hat{\psi}_{m}(\vartheta)=c_{1}^{m} e^{t_{1}^{m} \vartheta}+c_{2}^{m} e^{t_{2}^{m} \vartheta}+c_{3}^{m} e^{t_{3}^{m} \vartheta}+c_{4}^{m} e^{t_{4}^{m} \vartheta} \quad \forall m
$$

where the coefficients $c_{1}^{m}, c_{2}^{m}, c_{3}^{m}, c_{4}^{m}$ are determined by the boundary conditions. Imawaki and Takano (1974) obtained those numerical solutions (15) with and without the bottom friction and showed good agreement with their laboratory experiments. Their approach works fine and easily allows to include other effects (or terms) in their formulation, such as the aforementioned bottom friction and the lateral friction other than harmonic (or Laplacian) viscosity, so long as the variations of colatitudes are relatively small, that is, relatively narrow width of the zonal band of a polar cap $\left(\vartheta_{\mathrm{b}}=20^{\circ}\right.$ in their application).

Here, we solve the ordinary differential equations (10), without the above limitation, by making use of the associated Legendre functions of complex degrees $\mu$ and integer orders $m$ for real $x \in(-1,1)$ defined by

$$
\begin{aligned}
& P_{\mu}^{m}(x)=\frac{1}{2^{m} m !} \frac{\Gamma(\mu+1+m)}{\Gamma(\mu+1-m)}\left(1-x^{2}\right)^{\frac{m}{2}} \\
& \cdot F\left(-\mu+m, \mu+1+m, 1+m ; \frac{1-x}{2}\right),
\end{aligned}
$$

where $\Gamma(z)$ is the gamma function,

$$
\Gamma(z)=\int_{0}^{\infty} t^{z-1} e^{-t} d t \quad \text { for } \operatorname{Re}[z]>0
$$

$F(\alpha, \beta, \gamma ; x)$ is the Gauss hypergeometric function,

$$
F(\alpha, \beta, \gamma ; x)=\sum_{n=0}^{\infty} \frac{(\alpha)_{n}(\beta)_{n}}{(\gamma)_{n}} \frac{x^{n}}{n !} \text { for } x \in(-1,1)
$$


and $(\alpha)_{n}$ is defined by $(\alpha)_{0}=1$ and $(\alpha)_{n}=\alpha(\alpha+1)(\alpha+2) \cdots(\alpha+n-1)$ for $n \geq 1$ (see, for example, Abramowitz and Stegun, 1970). The associated Legendre functions (16) have the following properties:

$$
D_{m} P_{\mu}^{m}(\cos \vartheta)=-\mu(\mu+1) P_{\mu}^{m}(\cos \vartheta)
$$

and

$$
P_{-\mu-1}^{m}(\cos \vartheta)=P_{\mu}^{m}(\cos \vartheta) .
$$

By assuming that the solution is proportional to the associated Legendre function, $\hat{\psi}_{m}(\vartheta) \propto P_{\mu}^{m}(\cos \vartheta)$, and using the property (19), the ordinary differential equation (10) determines the allowable degrees $\mu$ for each given order $m$,

$$
-\mu(\mu+1)-k_{m}^{2}=0, \quad \text { or } \quad-\mu(\mu+1)+k_{m}^{2}=0 .
$$

The former quadratic equation allows two values of degrees $\mu=\mu_{m}^{-},-\mu_{m}^{-}-1$, where

$$
\mu_{m}^{-}=\frac{-1+\sqrt{1-4 k_{m}^{2}}}{2}
$$

By the property (20), however, only the $\mu=\mu_{m}^{-}$gives a linearly independent solution. Similarly, from the latter equation in Eq. (21) only the $\mu=\mu_{m}^{+}$, where

$$
\mu_{m}^{+}=\frac{-1+\sqrt{1+4 k_{m}^{2}}}{2},
$$

gives another linearly independent solution. The equation (10) is fourth order; the other two solutions $Q_{\mu_{m}^{ \pm}}^{m}(\cos \vartheta)$ of the associated Legendre functions of the second kind, which are divergent at the north pole $\vartheta=0$, are discarded.

Superposing the two linearly independent solutions $P_{\mu_{m}^{ \pm}}^{m}(\cos \vartheta)$ multiplied by $e^{i m \varphi}$ and summing over all orders $m$ in Eq. (8), we obtain the analytic solution of the stream function as

$$
\psi(\vartheta, \varphi)=\sum_{m=-\infty}^{\infty}\left[C_{m}^{-} P_{\mu_{m}^{-}}^{m}(\cos \vartheta)+C_{m}^{+} P_{\mu_{m}^{+}}^{m}(\cos \vartheta)\right] e^{i m \varphi} .
$$

Here, the coefficients $C_{m}^{ \pm}$are determined by the boundary conditions. We impose the no-slip condition at the spherical section of a fixed solid boundary except an inlet and an outlet,

$$
\psi\left(\vartheta_{\mathrm{b}}, \varphi\right)=\psi^{\mathrm{b}}(\varphi), \quad \frac{\partial \psi}{\partial \vartheta}\left(\vartheta_{\mathrm{b}}, \varphi\right)=0
$$

where $\psi^{\mathrm{b}}(\varphi)$ is a stream function prescribed at the boundary. Then, the coefficients are determined by

$$
\begin{gathered}
C_{0}^{-}+C_{0}^{+}=\hat{\psi}_{0}^{\mathrm{b}}, \\
C_{m}^{ \pm}=\mp \frac{1}{W_{m}}\left[\frac{d}{d x} P_{\mu_{m}^{\mp}}^{m}(x)\right]_{x=\cos \vartheta_{\mathrm{b}}} \hat{\psi}_{m}^{\mathrm{b}}=\mp \frac{1}{W_{m}} P_{\mu_{m}^{\mp}}^{m^{\prime}}\left(\cos \vartheta_{\mathrm{b}}\right) \hat{\psi}_{m}^{\mathrm{b}} \quad \forall m \neq 0,
\end{gathered}
$$


where $\hat{\psi}_{m}^{\mathrm{b}}$ are the Fourier coefficients of the $\psi^{\mathrm{b}}(\varphi)$ computed by using Eq. (9), and $W_{m}$ is defined by

$$
W_{m}=P_{\mu_{m}^{-}}^{m}\left(\cos \vartheta_{\mathrm{b}}\right) P_{\mu_{m}^{+}}^{{ }^{\prime}}\left(\cos \vartheta_{\mathrm{b}}\right)-P_{\mu_{m}^{+}}^{m}\left(\cos \vartheta_{\mathrm{b}}\right) P_{\mu_{m}^{-}}^{{ }^{\prime}}\left(\cos \vartheta_{\mathrm{b}}\right)
$$

We employ the parameters same as those in Imawaki and Takano (1974) to mimic circulations in the Arctic Ocean:

$$
\vartheta_{\mathrm{b}}=20^{\circ}, \quad r=6371 \mathrm{~km}, \quad|\boldsymbol{\Omega}|=7.292 \times 10^{-5} \mathrm{~s}^{-1}, \quad \nu=1.0 \times 10^{4} \mathrm{~m}^{2} \mathrm{~s}^{-1} .
$$

The horizontal Ekman number (7) is given by

$$
E_{\mathrm{H}}=1.689 \times 10^{-6} \text {. }
$$

\section{Results}

\subsection{Eigenfunctions}

The solution (24) is considered as superposition of the trigonometric eigenfunctions for the associated eigenvalues $k_{m}^{4}$ over all integers $m$,

$$
\psi(\vartheta, \varphi)=\hat{\psi}_{0}^{\mathrm{b}}+\sum_{m=1}^{\infty}\left(2 \operatorname{Re}\left[\hat{\psi}_{m}(\vartheta)\right] \cos m \varphi-2 \operatorname{Im}\left[\hat{\psi}_{m}(\vartheta)\right] \sin m \varphi\right)
$$

where the Fourier coefficients $\hat{\psi}_{m}(\vartheta)$ are given by

$$
\hat{\psi}_{m}(\vartheta)=C_{m}^{-} P_{\mu_{m}^{-}}^{m}(\cos \vartheta)+C_{m}^{+} P_{\mu_{m}^{+}}^{m}(\cos \vartheta)
$$

Fig. 2 shows contours (or streamlines) of the lowest four sinusoidal eigenfunctions,

$$
\psi(\vartheta, \varphi)=-2 \operatorname{Im}\left[\hat{\psi}_{m}(\vartheta)\right] \sin m \varphi \quad \text { for } 1 \leq m \leq 4,
$$

the solutions (24) with the prescribed stream functions (25) at the boundary,

$$
\psi^{\mathrm{b}}(\varphi)=\sin m \varphi \quad \text { for } 1 \leq m \leq 4
$$

indicated in the upper plot of each figure (a)-(d). The contour interval is 0.2 between -0.8 and 0.8 , and the polar cap is viewed from the north pole.

The first sinusoidal eigenfunction shows that the flow comes into the polar cap from the right half of the boundary and goes away from the other half in Fig. 2(a). The inflow turns westward immediately after entering the polar cap and separates into two branches: one is cyclonic and the other is anticyclonic flows along the boundary. These two branches merge again and turn eastward 
before exiting the polar cap. The streamline at the center of the inflow passes through the north pole and the center of the outflow; this line divides the inflow into the above two branches. As described below Eq. (3), the fluid particles move nearly along lines of constant latitudes in the geostrophic balance everywhere except near the north pole and very close to the boundary where the flow is relatively slow and the viscous force dominates over the Coriolis force.

The second sinusoidal eigenfunction shows that the flow comes into the polar cap from the right and left quarters centered at the longitudes $0, \pi$ of the boundary and goes away from the other two quarters in Fig. 2(b). The flow field has twofold symmetry about the rotation axis. The inflow turns westward immediately after entering the polar cap and separates into cyclonic and anticyclonic flows along the boundary. The cyclonic/anticyclonic flow merges with the anticyclonic/cyclonic branch of the other inflow and turns eastward before exiting the polar cap. The streamlines at the centers of the inflows spirally go into and out of the north pole, and pass through the centers of the outflows; these lines divide the inflows into the above a couple of two branches. The north pole is a stagnation point in the flow field. The fluid particles move nearly along lines of constant latitudes in the geostrophic balance everywhere except near the north pole and very close to the boundary.

The flow fields of the third and fourth sinusoidal eigenfunctions in Fig. 2(c,d) are similar to that of the second eigenfunction but have threefold and fourfold symmetries about the rotation axis, respectively.

\subsection{Analytic Solution}

We prescribe the stream function $\psi^{\mathrm{b}}(\varphi)$ in Eq. (25) on the boundary as

$$
\psi^{\mathrm{b}}(\varphi)=A \times \begin{cases}\frac{\varphi}{\varphi_{1}} & \text { for } 0 \leq \varphi<\varphi_{1}, \\ 1 & \text { for } \varphi_{1} \leq \varphi<\varphi_{2}, \\ 1-\frac{\varphi-\varphi_{2}}{\varphi_{3}-\varphi_{2}} & \text { for } \varphi_{2} \leq \varphi<\varphi_{3}, \\ -\frac{\varphi-\varphi_{3}}{\varphi_{4}-\varphi_{3}} & \text { for } \varphi_{3} \leq \varphi<\varphi_{4}, \\ -1 & \text { for } \varphi_{4} \leq \varphi<\varphi_{5}, \\ -1+\frac{\varphi-\varphi_{5}}{2 \pi-\varphi_{5}} & \text { for } \varphi_{5} \leq \varphi<2 \pi,\end{cases}
$$


where $A$ is a constant amplitude and $\varphi_{i}(i=1,2,3,4,5)$ are constant longitudes. There are an inlet between $\varphi_{5}$ and $\varphi_{1}$, and an outlet between $\varphi_{2}$ and $\varphi_{4}$ (see Fig. 3). The Fourier coefficients $\hat{\psi}_{m}^{\mathrm{b}}$ are computed by utilizing Eq. (9) if $m=0$,

$$
\hat{\psi}_{0}^{\mathrm{b}}=\frac{A}{2 \pi}\left[\frac{\varphi_{1}}{2}+\left(\varphi_{2}-\varphi_{1}\right)+\frac{\varphi_{3}-\varphi_{2}}{2}-\frac{\varphi_{4}-\varphi_{3}}{2}-\left(\varphi_{5}-\varphi_{4}\right)-\frac{2 \pi-\varphi_{5}}{2}\right],
$$

otherwise,

$\hat{\psi}_{m}^{\mathrm{b}}=\frac{A}{2 \pi m^{2}}\left[\frac{e^{-i m \varphi_{1}}-1}{\varphi_{1}}-\frac{e^{-i m \varphi_{3}}-e^{-i m \varphi_{2}}}{\varphi_{3}-\varphi_{2}}-\frac{e^{-i m \varphi_{4}}-e^{-i m \varphi_{3}}}{\varphi_{4}-\varphi_{3}}+\frac{1-e^{-i m \varphi_{5}}}{2 \pi-\varphi_{5}}\right]$.

We assume uniform flow within the inlet and outlet, that is, $\varphi_{1}=2 \pi-\varphi_{5}\left(=\varphi^{\mathrm{i}}\right)$ and $\varphi_{3}-\varphi_{2}=\varphi_{4}-\varphi_{3}\left(=\varphi^{\circ}\right)$, where $\varphi^{\mathrm{i}}, \varphi^{\mathrm{o}}$ are half of the central angles (i.e., the inscribed angles) of the inlet and outlet, respectively,

$$
\hat{\psi}_{m}^{\mathrm{b}}=A \times \begin{cases}\frac{\left(\varphi_{2}-\varphi_{1}\right)-\left(\varphi_{5}-\varphi_{4}\right)}{2 \pi}=\frac{\varphi_{3}-\pi}{\pi} & \text { if } m=0, \\ \frac{-i}{\pi m^{2}}\left[\frac{\sin m \varphi^{\mathrm{i}}}{\varphi^{\mathrm{i}}}-e^{-i m \varphi_{3}} \frac{\sin m \varphi^{\mathrm{o}}}{\varphi^{\mathrm{o}}}\right] & \text { otherwise. }\end{cases}
$$

Eq. (36) describes the mean zonal flow vanishes if the centers of the inlet and outlet make the straight angle. For simplicity's sake, we consider this case, that is, the centers of the inlet and outlet are oppositely placed exactly, i.e., $\varphi_{3}=\pi$, then Eq. (36) is simplified as

$$
\hat{\psi}_{m}^{\mathrm{b}}= \begin{cases}0 & \text { if } m=0, \\ \frac{-i A}{\pi m^{2}}\left[\frac{\sin m \varphi^{\mathrm{i}}}{\varphi^{\mathrm{i}}}-(-1)^{m} \frac{\sin m \varphi^{\mathrm{o}}}{\varphi^{\mathrm{o}}}\right] & \text { otherwise. }\end{cases}
$$

Moreover, we assume that the widths of the inlet and outlet are equal, i.e., $\varphi^{\mathrm{i}}=\varphi^{\mathrm{o}}$, then

$$
\hat{\psi}_{m}^{\mathrm{b}}= \begin{cases}0 & \text { for } m=0, \pm 2, \pm 4, \ldots, \\ \frac{-2 i A \sin m \varphi^{\mathrm{i}}}{\pi m^{2} \varphi^{\mathrm{i}}} & \text { for } m=1, \pm 3, \pm 5, \ldots\end{cases}
$$

Substituting Eq. (38) into Eq. (26) and then Eq. (24), we obtain the analytic solution of steady Stokes flow on a rotating polar cap for the prescribed stream function Eq. (34) at the boundary.

Fig. 4 shows contours (or streamlines) of the stream function together with the prescribed stream function on the boundary (upper plot). The contour interval is 0.2 between -0.8 and 0.8 , and the polar cap is viewed from the north pole. The inscribed angles $\varphi^{\mathrm{i}}, \varphi^{\circ}$ of the inlet and outlet are both set to $10^{\circ}$, 
and the amplitude $A$ is set to 1 . The infinite series in Eq. (24) is truncated at the wave number $M= \pm 60$. The flow field is quite similar to that of the first sinusoidal eigenfunction in Fig. 2(a) except near the boundary. The flow comes into the polar cap from the inlet and goes away from the outlet in Fig. 4. The inflow turns westward immediately after entering the polar cap and separates into cyclonic and anticyclonic flows along the boundary. These two branches merge again and turn eastward before exiting the polar cap. The streamline at the center of the inflow passes through the north pole and the center of the outflow; this line divides the inflow into the above two branches. The fluid particles move nearly along lines of constant latitudes in the geostrophic balance everywhere except near the north pole. Those westward/eastward flow near the inlet/outlet as well as concentrated flow near the boundary can be interpreted in terms of the dissipating planetary waves (Sakai and Imawaki, 1981) - the Rossby-Haurwitz waves (Rossby, 1939; Haurwitz, 1940) with dissipation.

To see the balance of the forces in Eq. (1), we draw the Coriolis force (arrows) and fluid speed (red color) in Fig. 5(a), and the pressure gradient force (arrows) and relative vorticity (red denotes cyclonic vorticity, while blue anticyclonic) in Fig. 5(b). The length of each arrow represents the magnitude of each vector. The Coriolis force is directed to the right of the motion, that is, away from/towards the north pole on the cyclonic/anticyclonic flow. On the other hand, the pressure gradient force is directed towards/away from the north pole on the cyclonic/anticyclonic flow, for the cyclonic/anticyclonic (or relatively low/high pressure) branch along the boundary generates anticyclonic/cyclonic (or relatively high/low pressure) vorticity very close to the boundary. These two forces are in geostrophic balance on the above two branches, where the flow is relatively rapid and the Coriolis force dominates over the viscous force.

Finally, we look into dependence of flow field upon the horizontal Ekman number $E_{\mathrm{H}}$ in Eq. (7). Fig. 6 shows streamlines for $E_{\mathrm{H}}=$ (a) 1.0 , (b) $1.0 \times 10^{-4}$, (c) $1.0 \times 10^{-5}$, (d) $5.0 \times 10^{-6}$, (e) $1.689 \times 10^{-6}$, (f) $5.0 \times 10^{-7}$. Fig. 6(e) is the analytic solution in Fig. 4(b). In the largest horizontal Ekman number (a), the viscous force dominates over the Coriolis force. The flow field is quite similar to one without the rotation of the polar cap in Fig. 6(a). The fluid particles cross lines of constant latitudes everywhere. On the other limit, in the smallest horizontal Ekman number (f), the Coriolis force dominates over the viscous force. The flow field is quite similar to that of the analytic solution in Fig. 6(e), but more strongly constrained by the geostrophic balance in Fig. 6(f). The fluid particles move nearly along lines of constant latitudes everywhere except near the north pole. In the moderate horizontal Ekman number (b)-(e), the viscous and Coriolis forces compete with each other to make the flow field a mixture of those in the extreme cases (a) and (f) in Fig. 6(b)-(e), respectively.

Direct comparison of our results with circulation in the Arctic Ocean is difficult 
since other factors - such as non-linearity, wind stress, density stratification, bottom topography, and sea ice - significantly affect the real ocean circulation. Our results may be considered as one of the underlying building blocks for the circulation in the Arctic. Here, we note a crude application of our solution to the Arctic Ocean. The volume transport - total inflow per unit time - through the Bering Strait into the Arctic Ocean is estimated about $0.8 \mathrm{~Sv}$ (Sverdrup, $1 \mathrm{~Sv}=10^{6} \mathrm{~m}^{3} / \mathrm{s}$ ) by Woodgate and Aagaard (2005). On the other hand, the volume transport - total outflow per unit time - through the Fram Strait from the Arctic Ocean is estimated about 4.2 Sv by Fahrbach et al. (2001). Suppose that the Bering Strait is $100 \mathrm{~km}$ width and $50 \mathrm{~m}$ depth, the Fram Strait is 300 $\mathrm{km}$ width and $2000 \mathrm{~m}$ depth, and the inflow and outflow are uniform. Then, the fluid speeds through the Bering and Fram Straits are estimated about $16 \mathrm{~cm} / \mathrm{s}$ and $0.7 \mathrm{~cm} / \mathrm{s}$, respectively. Using these fluid speeds as the boundary conditions, recently, Taniguchi and Yamada (private communication) study an unsteady, non-linear regime of our analytic solution by a direct numerical simulation. Their result suggests that our steady, linear solution is stable due to relatively slow inflow and outflow.

\section{Concluding Remarks}

We obtained an analytic solution of steady Stokes flow, that is, two-dimensional, steady, linear, viscous flow on a rotating polar cap. The solution of the stream function is expressed as the Fourier series in longitudes and the associated Legendre functions of complex degrees in cosines of colatitudes. In a relatively small horizontal Ekman number $E_{\mathrm{H}} \ll 1$, the fluid particles move nearly along lines of constant latitudes in the geostrophic balance everywhere except near the north pole. On the other hand, in a relatively large horizontal Ekman number $E_{\mathrm{H}} \sim 1$, the fluid particles cross lines of constant latitudes everywhere. These results support the approximation analysis and laboratory experiment studied by Imawaki and Takano (1974).

Our formulation includes full sphericity; therefore, we may extend the domain of a polar cap by increasing the boundary colatitude $\vartheta_{\mathrm{b}}$, which is invalid in the approximation made by Imawaki and Takano (1974). Fig. 7, for example, shows streamlines for $\vartheta_{\mathrm{b}}=45^{\circ}$ and $E_{\mathrm{H}}=5.0 \times 10^{-6}$. Our formulation successfully computes the stream function even for the larger colatitude of the boundary. Our analytic solution is also applicable to steady Stokes flow on a rotating spherical segment - region of a sphere which lies between a pair of given parallel planes - by introducing the associated Legendre functions of the second kind as described below Eq. (23) in Section 2.

In this paper we studied an analytic solution on a rotating polar cap. We are now curious about stability of our steady, linear solution with respect to 
the inflow and outflow as well as the flow patterns in an unsteady, non-linear regime for a better understanding of the system. Our preliminary results show that a stationary solution is stable up to the relatively large inflow and outflow in the real Arctic Ocean. If the inflow and outflow are further increased, a steady, non-linear solution becomes unstable with a Hopf bifurcation to an oscillatory solution. These results will be reported in near future.

\section{Acknowledgments}

The author HK would like to express his cordial gratitude to Dr. Harper L. Simmons for bringing this problem and fruitful discussions. The HK also would like to thank Dr. David Walsh for his valuable comments on this study. Dr. Yuki Taniguchi and Prof. Michio Yamada stimulated the HK through fruitful discussions.

We are indebted to two anonymous referees and Editor Yoshi-Yuki Hayashi for valuable comments and patience during the review process. A special thanks goes to Editor-in-Chief Shigeo Kida for his valuable suggestion to proceed to the final revision.

\section{References}

Abramowitz, M., Stegun, I.A., 1970. Handbook of Mathematical Functions with Formulas, Graphs, and Mathematical Tables. Dover Publications, Inc., New York.

Courant, R., Hilbert, D., 1989. Methods of Mathematical Physics, Volume 1. John Wiley \& Sons, New York, 307-318.

Fahrbach, E., Meincke, J., Østerhus, S., Rohardt, G., Schauer, U., Tverberg, V., Verduin, J., 2001. Direct measurements of volume transports through Fram Strait. Polar Res. 20(2), 217-224.

Haurwitz, B., 1940. The motion of atmospheric disturbances on the spherical earth. J. Mar. Res. 3, 254-267.

Imawaki, S., Takano, K., 1974. Planetary flow in a circular basin. Deep-Sea Res. 21, 69-77.

Pratt, L.J., 1997. Hydraulically drained flows in rotating basins. Part II: Steady flow. J. Phys. Oceanogr. 27, 2522-2535.

Rossby, C.G., 1939. Relation between variations in the intensity of the zonal circulation of the atmosphere and the displacements of the semi-permanent centers of action. J. Mar. Res. 2, 38-55.

Sakai, S., Imawaki, S., 1981. Notes on linear and nonlinear barotropic flows in a polar circular basin. J. Oceanogr. Soc. Japan 37, 287-293. 
Woodgate, R.A., Aagaard, K., 2005. Revising the Bering Strait freshwater flux into the Arctic Ocean. Geophys. Res. Lett. 32, L02602, doi:10.1029/2004GL021747.

Yang, J., 2005. The Arctic and subarctic ocean flux of potential vorticity and the Arctic Ocean circulation. J. Phys. Oceanogr. 35, 2387-2407. 


\section{Figure Captions}

Fig. 1: Configuration of a polar cap.

Fig. 2: Streamlines of the (a) first, (b) second, (c) third, and (d) fourth sinusoidal eigenfunctions. The upper plot in each figure shows the prescribed stream function at the boundary.

Fig. 3: Stream function prescribed on the boundary.

Fig. 4: (a) Prescribed stream function at the boundary. (b) Streamlines of the steady Stokes flow on a rotating polar cap.

Fig. 5: (a) Coriolis force (arrows) and fluid speed. The denser the red is, the faster the flow is. (b) Pressure gradient force (arrows) and relative vorticity. Red denotes cyclonic vorticity, while blue anticyclonic vorticity. The length of each arrow represents the magnitude of each vector.

Fig. 6: Streamlines for the horizontal Ekman number $E_{\mathrm{H}}=$ (a) 1.0, (b) $1.0 \times 10^{-4}$, (c) $1.0 \times 10^{-5}$, (d) $5.0 \times 10^{-6}$, (e) $1.689 \times 10^{-6}$, (f) $5.0 \times 10^{-7}$.

Fig. 7: Streamlines for the boundary colatitude $\vartheta_{\mathrm{b}}=45^{\circ}$ and the horizontal Ekman number $E_{\mathrm{H}}=5.0 \times 10^{-6}$. 


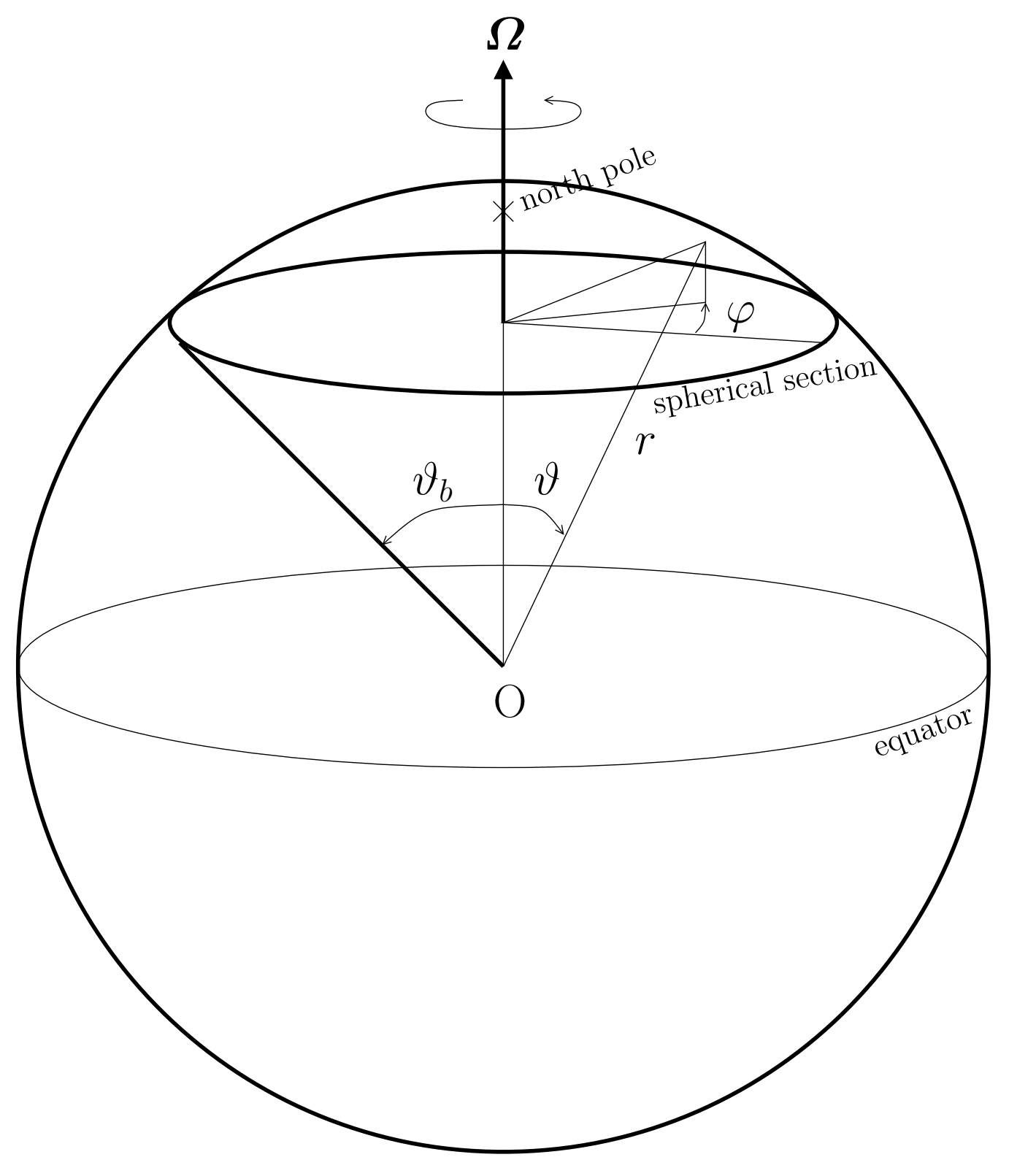

Fig. 1: H. Kitauchi and M. Ikeda. 
(a)
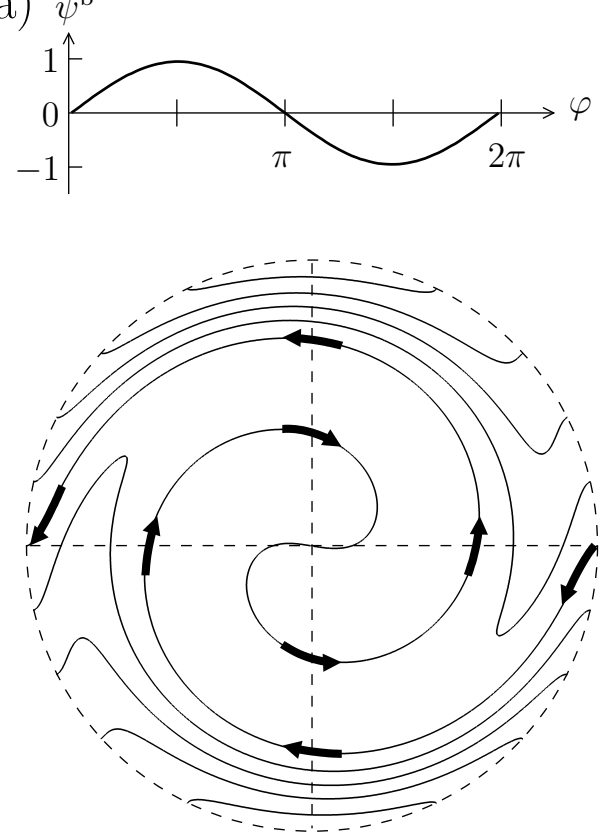

(b) $\psi^{\mathrm{b}}$
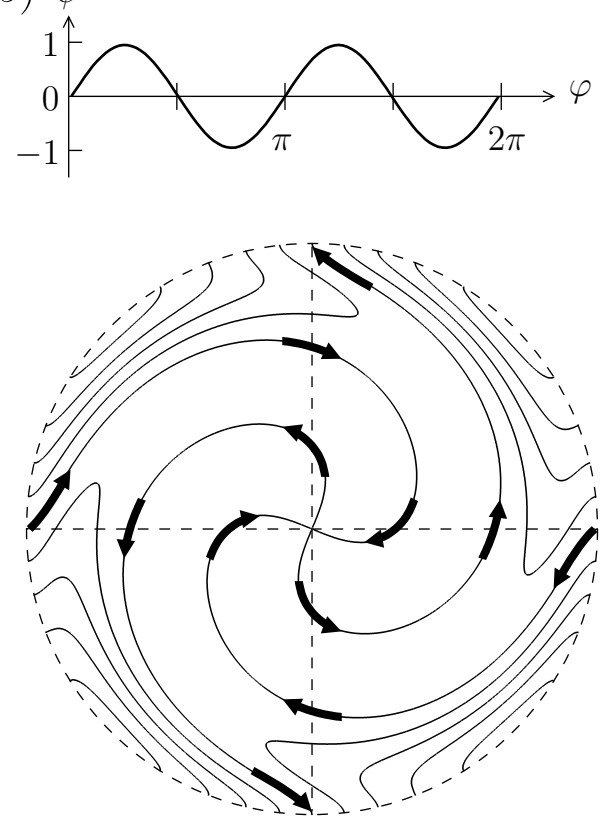

(c) $\psi^{\mathrm{b}}$
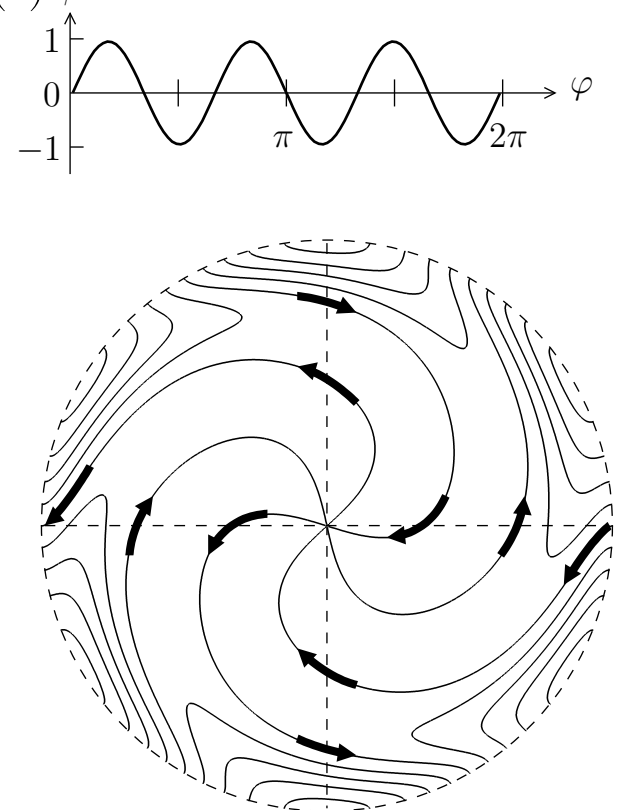

(d) $\psi^{\mathrm{b}}$
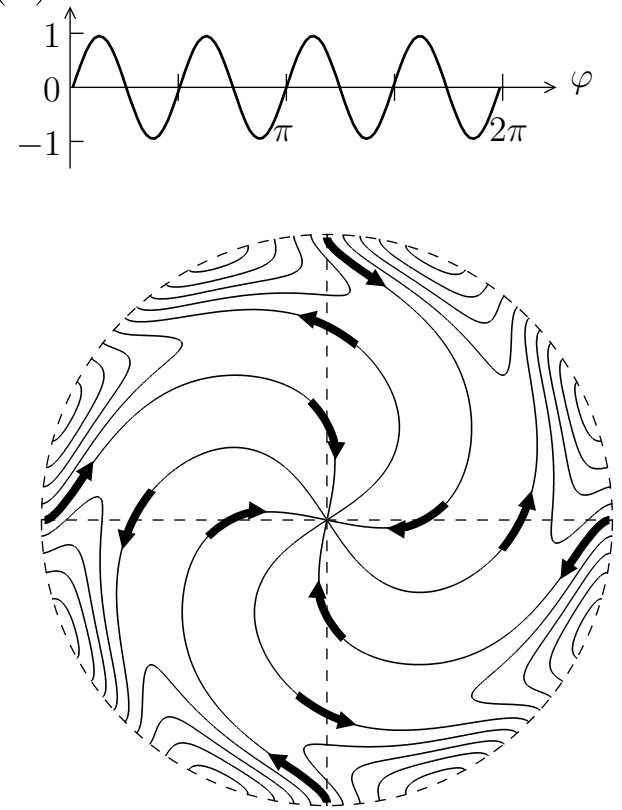

Fig. 2: H. Kitauchi and M. Ikeda. 


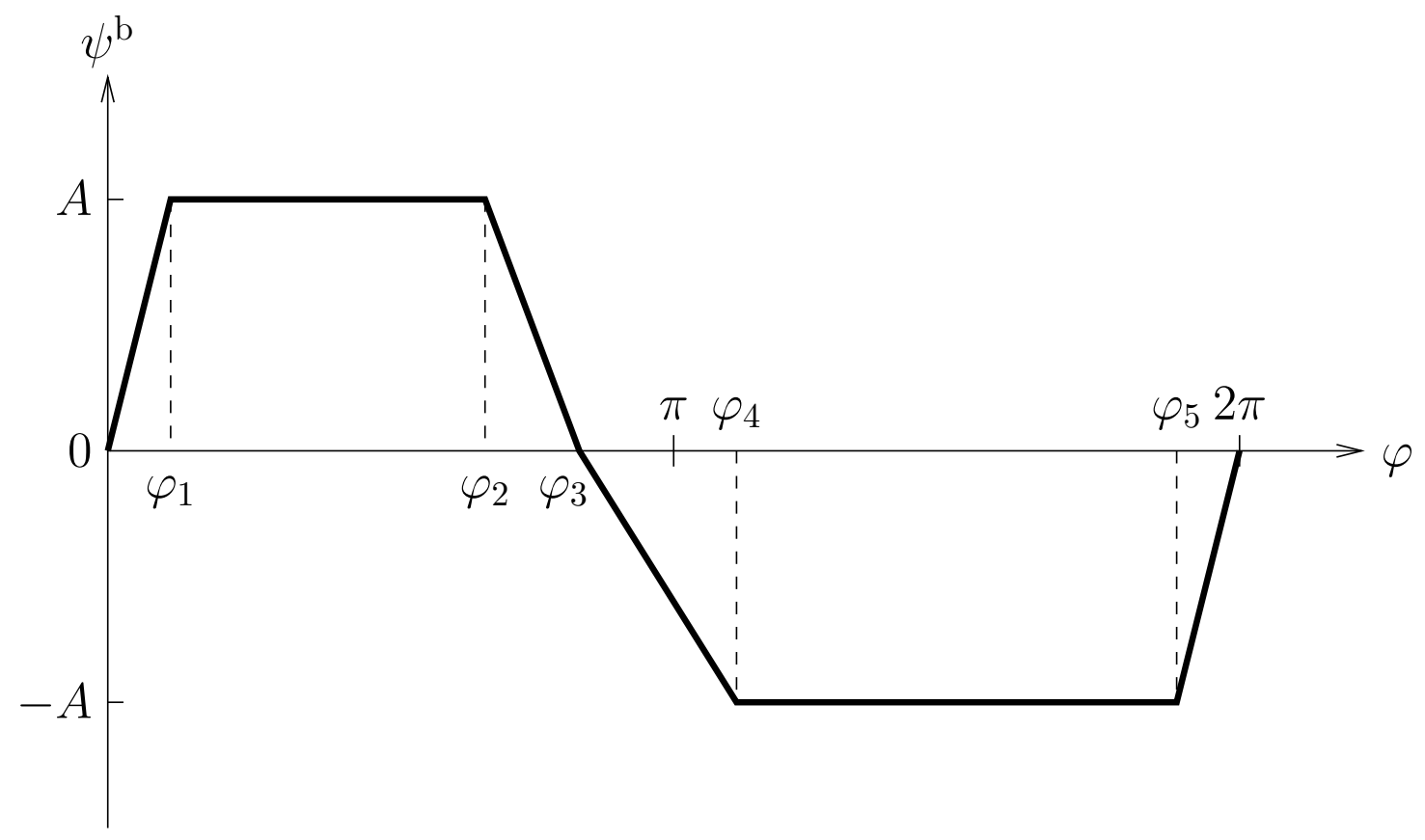

Fig. 3: H. Kitauchi and M. Ikeda. 


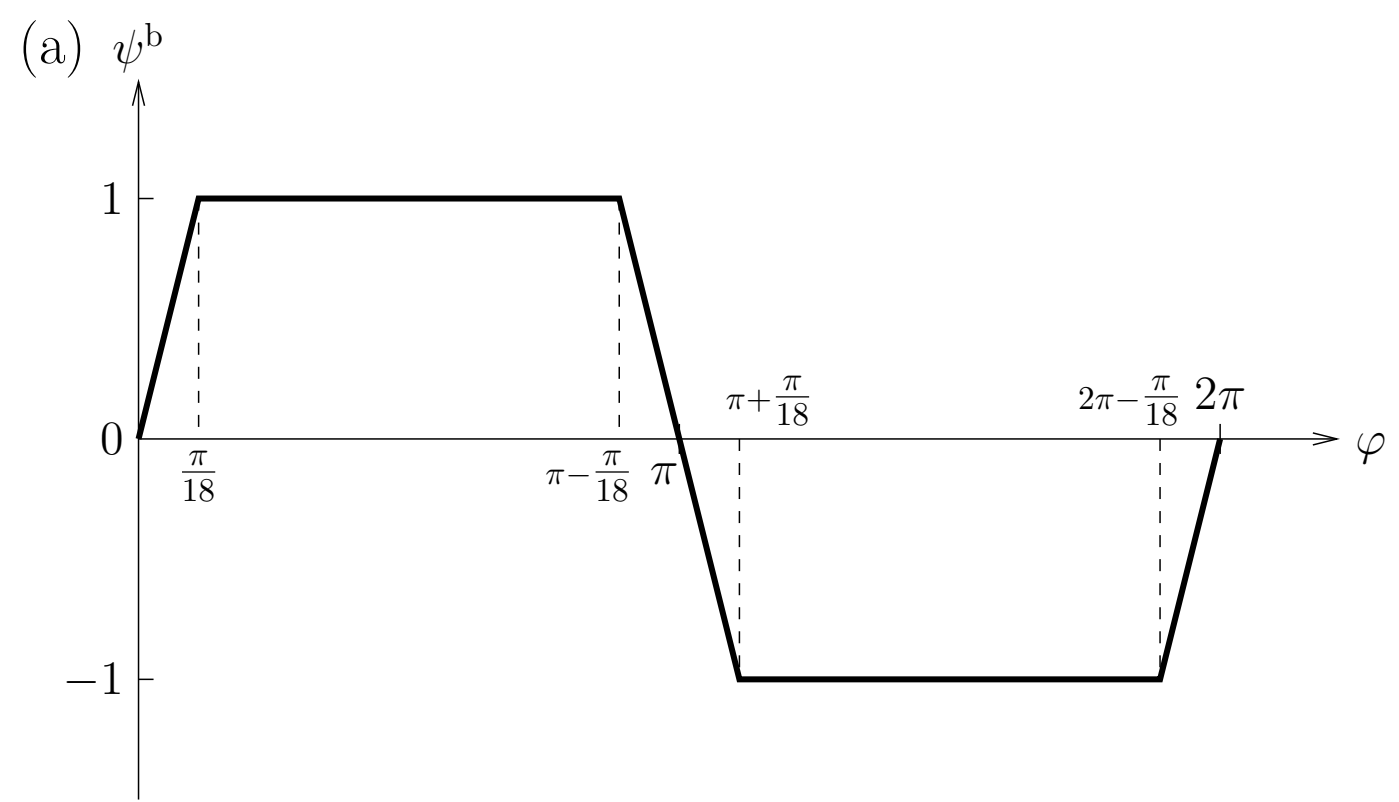

(b)

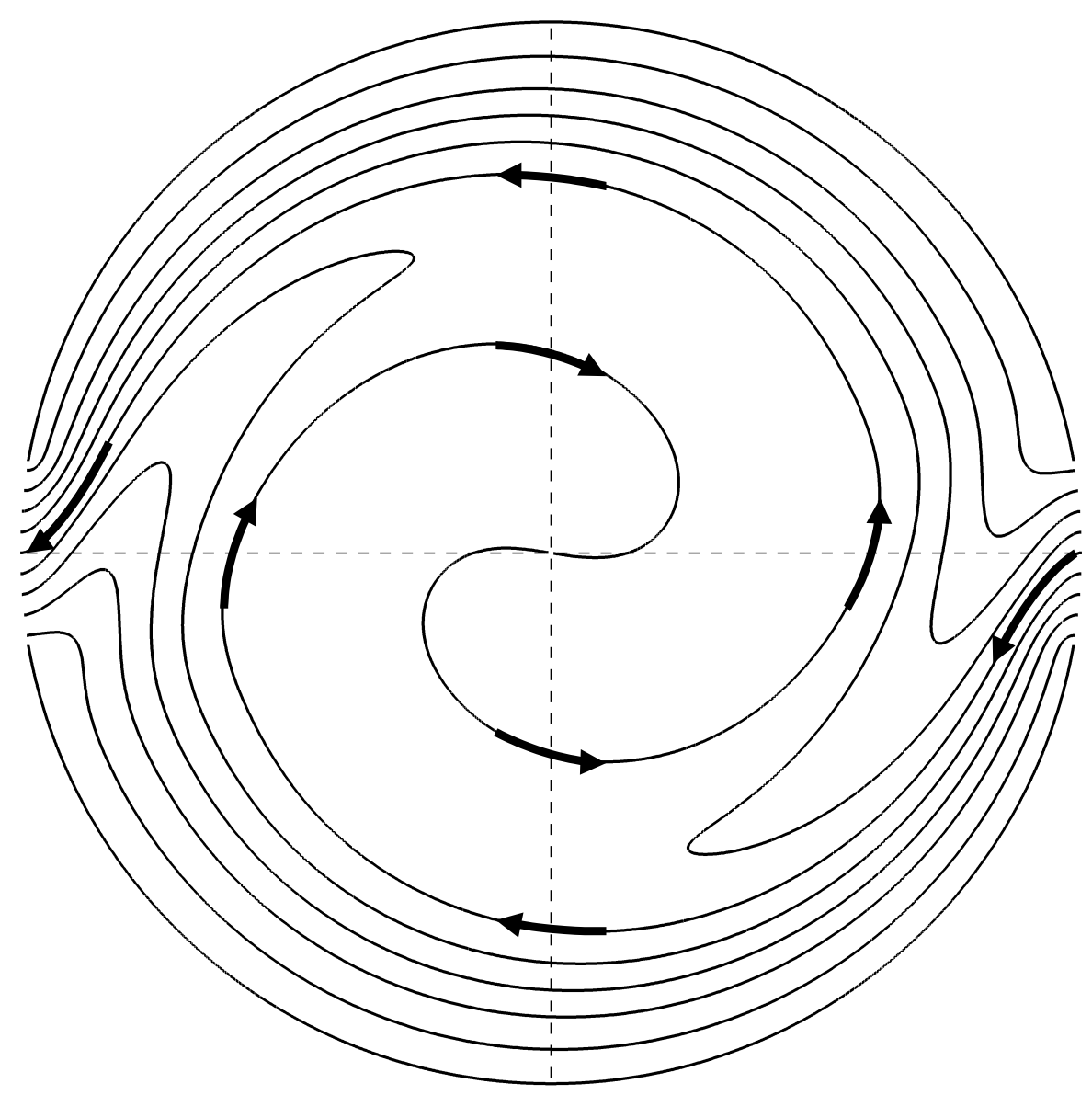

Fig. 4: H. Kitauchi and M. Ikeda. 

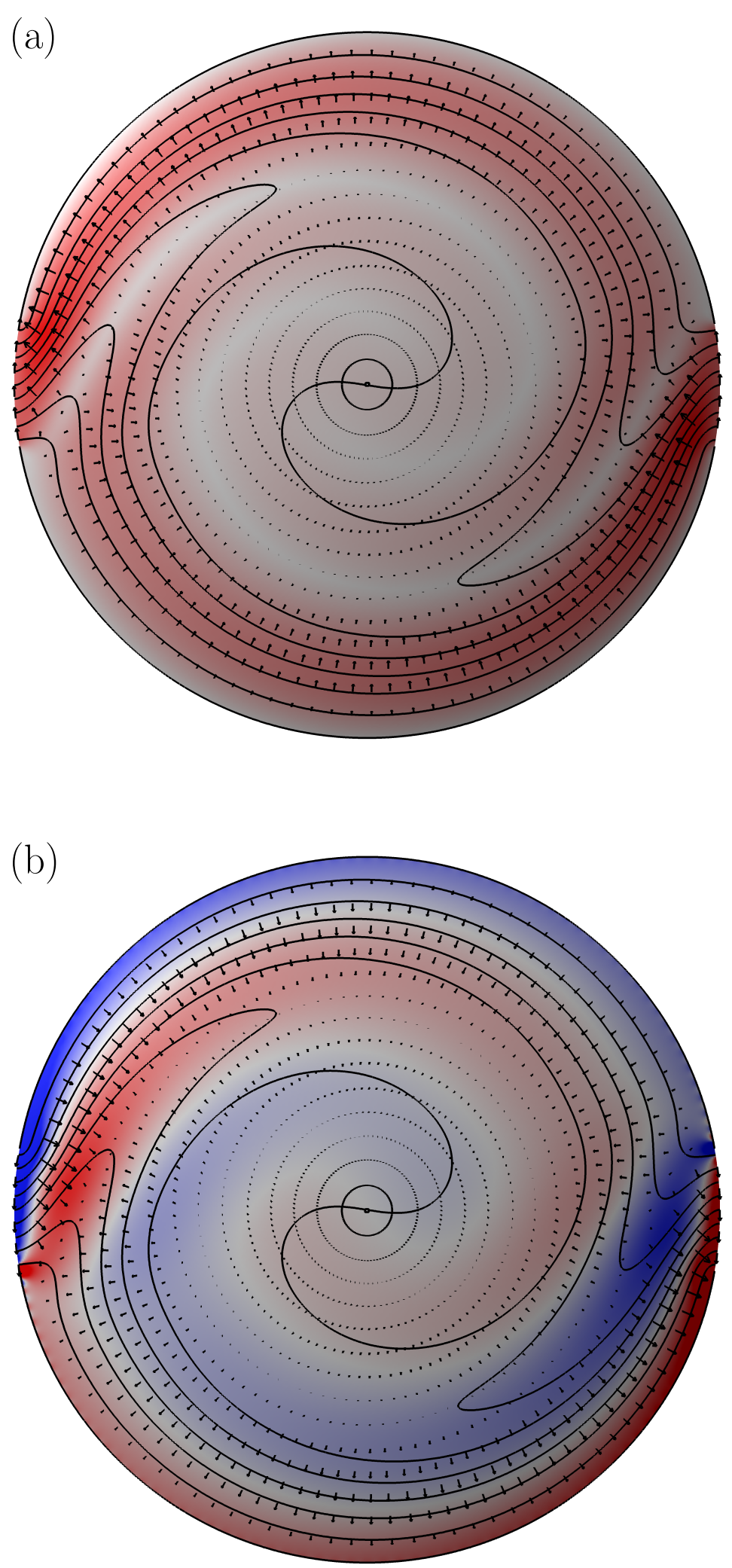

Fig. 5: H. Kitauchi and M. Ikeda. 

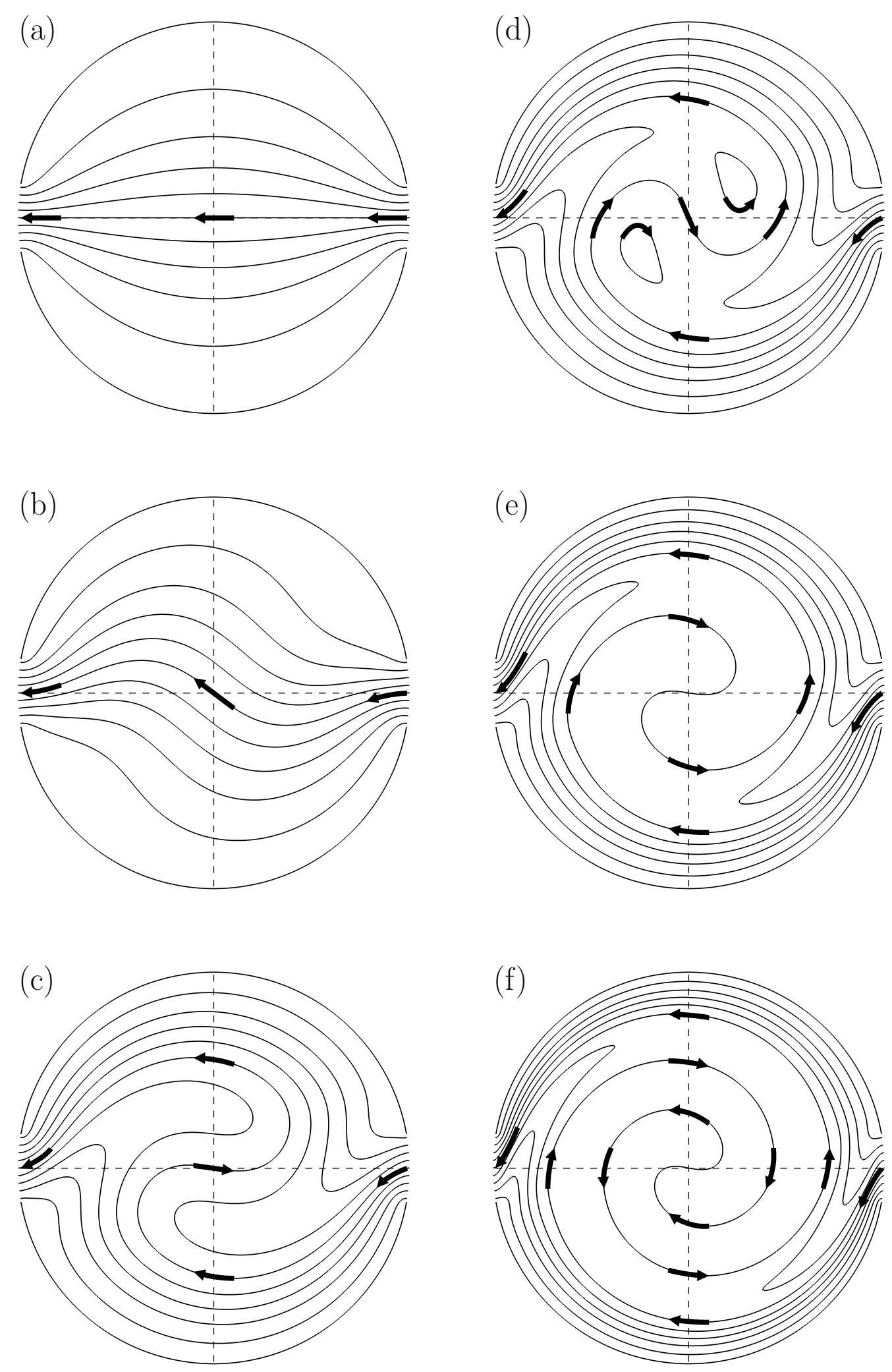

Fig. 6: H. Kitauchi and M. Ikeda. 


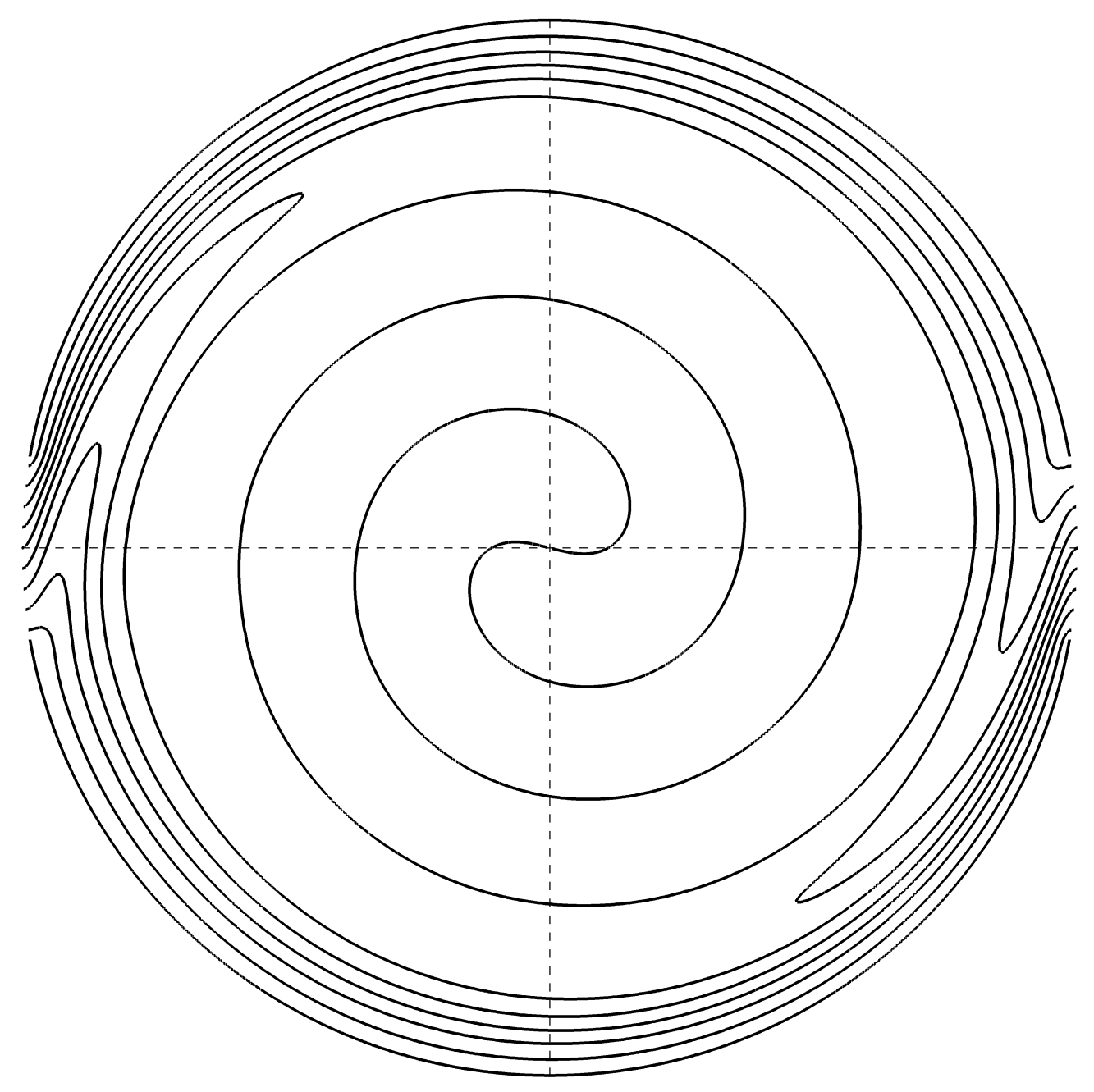

Fig. 7: H. Kitauchi and M. Ikeda. 